\title{
Investigation of field, carrier, and coherent phonon dynamics in low-temperature grown GaAs
}

\author{
T. Dekorsy and H. Kurz \\ Institute of Semiconductor Electronics, RWTH Aachen, D-52056 Aachen, Germany \\ X. Q. Zhou \\ Max-Planck-Insitut für Festkörperforschung, Heisenbergstr. 1, D-70506 Stuttgart 80, Germany \\ K. Ploog \\ Paul-Drude Institut für Festkörperphysik, Hausvogteiplatz 5-7, D-10117 Berlin, Germany
}

\begin{abstract}
We compare the dynamics of electric field, transport, and coherent phonons in as-grown and annealed low-temperature (LT) GaAs by an electro-optic technique on a subpicosecond time scale. The buildup and decay of space-charge fields associated with the photo-Dember effect are investigated. The recombination dynamics of trapped carriers is monitored via the ps decay of the electro-optic signal. Differences in annealed and as-grown LT GaAs are related to the different microscopic form of excess arsenic and point defect density. In the coherent phonon signal a large red shift of the LO phonon and an additional local vibration below the LO phonon provides information on structural defects in as-grown LT GaAs.
\end{abstract}

Low-temperature (LT) GaAs has recently attracted interest due to its application in electronic and optoelectronic devices. ${ }^{1-3}$ The main advantages of postgrowth annealed LT GaAs in electronic applications is its high resistivity. ${ }^{4}$ This electronic property is closely related to ultrashort mobile carrier lifetimes in the subpicosecond regime, while the carrier mobility remains relatively high.

LT GaAs is grown by molecular beam epitaxy (MBE) at temperatures of approximately $200^{\circ} \mathrm{C}$, resulting in an excess arsenic concentration of $1 \%-2 \%$. The microscopic form of the excess arsenic is still a point of discussion. One of the dominant point defects is an $\mathrm{As}_{\mathrm{Ga}}$ antisite at densities up to $10^{20} \mathrm{~cm}^{-3}$ depending of the exact growth parameters. Post-growth annealing of LT GaAs at temperatures above $600^{\circ} \mathrm{C}$ leads to the nucleation of excess arsenic in As precipitates. The influence of As precipitates on the high resistivity of anncaled LT GaAs is related to As clusters, which act as burried Schottky barriers responsible for trapping of electrons and holes. ${ }^{5}$ Although the mobile carrier lifetime can be determined from fs time-resolved luminescense upconversion ${ }^{6}$ or via the photoconductive response of LT GaAs based switches, ${ }^{7}$ the carrier lifetime in trapped states is hardly addressed by this and other techniques, such as deep level transient spectroscopy, photoluminescense, and photocurrent measurements. The determination of the recombination rate of carriers trapped in defects is of technological importance, since it basically determines the high frequency performance of LT GaAs based devices.

We apply reflective electro-optic sampling (REOS) with 2-eV laser pulses and a time resolution of approximately 50 fs for the investigation of LT GaAs. The REOS technique enables the direct and quantitative detection of electric field changes perpendicular to the samples surface via the linear electro-optic effect (Pockels effect) at (100) orientated GaAs. This method opens a new way for the material characterization of low-temperature grown III-V compounds. It enables the study of subpicosecond trans- port dynamics ${ }^{8}$ such as the photo-Dember effect, the investigation of structural defects via the coherent lattice vibration dynamics, ${ }^{9}$ and the determination of carrier lifetimes in trapped states. As-grown and postgrowth annealed LT GaAs has sufficient crystal quality to exhibit a Pockels effect, so that internal electric field changes can be detected by an all-optical method in a straightforward way with fs resolution. The experimental setup is described in Ref. 8 in detail.

The LT GaAs samples are grown by $\mathrm{MBE}$ at $200^{\circ} \mathrm{C}$. We compare as-grown with annealed LT GaAs $\left(600^{\circ} \mathrm{C}\right.$ anneal for $10 \mathrm{~min}$ ). The trapping time of mobile carriers has been determined by luminescence upconversion and correlation spectroscopy in the annealed sample to be 1 ps for electrons and holes. ${ }^{6}$

The REOS signals of annealed LT GaAs are depicted in Fig. 1 for two different excitation densities. The signals reveal a fast initial rise on the time scale of the pump pulse, which is caused by a fast initial screening of built-in electric fields. This ultrafast screening generates a burst of coherent phonons within the surface space-charge field, which are clearly observed as a periodic modulation via the associated macroscopic polarization. The amplitude of the initial field screening corresponds to maximum electric field

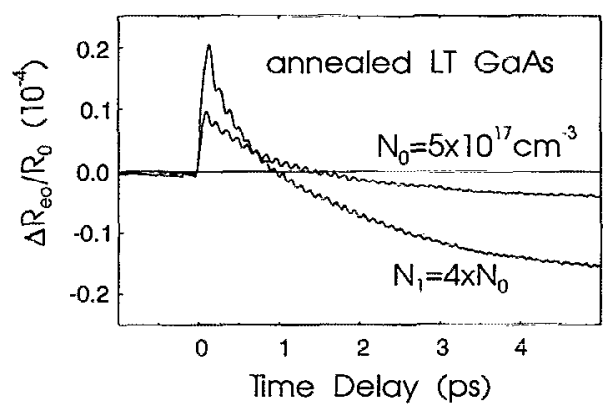

FIG. 1. REOS signals of annealed LT GaAs at the excitation densities of $5 \times 10^{17} \mathrm{~cm}^{-3}$ and $2 \times 10^{18} \mathrm{~cm}^{-3}$. 


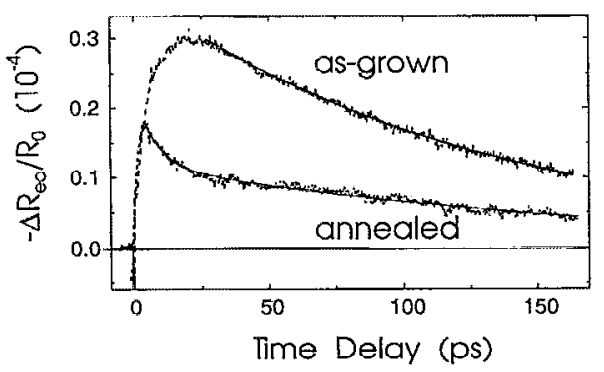

FIG. 2. Long delay time REOS measurements on annealed and as-grown LT GaAs (dashed curves) and numerical fits to the data (solid curves).

changes of about $20 \mathrm{kV} / \mathrm{cm}$. The fast decay and sign reversal within the first picosecond closely follows the buildup of a photo-Dember field, which is primarily determined by the difference in the mobilities between electrons and holes. ${ }^{10}$ This effect has not been observed in doped crystalline $\mathrm{GaAs}$ at comparable excitation densities since screening dominates the dynamics in the case of high electric surface fields. ${ }^{8}$ The experimental results at different excitation densities are in agreement with the theoretically expected contributions from screening and the buildup of a Dember field. Increasing the excitation densities by a factor 4 results in an increase of the initial field screening by 2 as expected from a $N^{1 / 2}$ dependence of screening below the saturation density. The Dember-field contribution scales linearily with the excitation density as expected from the theory. ${ }^{10}$ An additional confirmation for the buildup of a Dember field is obtained by additional continuous wave (cw) illumination of the sample. Under $\mathrm{cw}$ illumination the initial screening amplitude is reduced due to additional static screening, while the Dember-field contribution remains unchanged.

Figure 2 shows the signal decay on a picosecond time scale of the as-grown sample, which is clearly monoexponential with a time constant of $\tau=125 \pm 2 \mathrm{ps}$. The annealed sample, however, exhibits two time constants, $\tau_{1}=7 \pm 2 \mathrm{ps}$ and $\tau_{2}=160 \pm 10 \mathrm{ps}$ as determined from numerical fits to the data. These time constants are independent of the excitation density in the range of $5 \times 10^{17} \mathrm{~cm}^{-3}$ to $2 \times 10^{18}$ $\mathrm{cm}^{-3}$.

For the as-grown sample, we assume the $\mathrm{As}_{\mathrm{Ga}}$ antisites to be the most dominant point defects. ${ }^{11}$ Carriers in deep traps related to these point defects have emission rates into the conduction or valence bands in the order of $\mathrm{Hz}$ and can therefore be excluded for the interpretation of ps time constants. Further transport contributions are expected due to hopping between deep levels or a deep level conduction band. We relate the signal decay to the probability for the release of a carrier trapped at a deep level, which is proportional to $\exp \left(-\epsilon_{d} / k_{b} T+2 r / a\right)$. Here, $\epsilon_{d}$ is the mean energetic distance of deep levels, $r$ the localization radius of the defect wave function, and $a$ the mean spatial distance of the traps. ${ }^{12}$

The long time constant in both samples is related to carriers captured in $\mathrm{As}_{\mathrm{Ga}}$ antisites. The increase of the time constant in annealed LT GaAs reflects the decrease in the point dcfect density (increase in the average distance $a$ ),

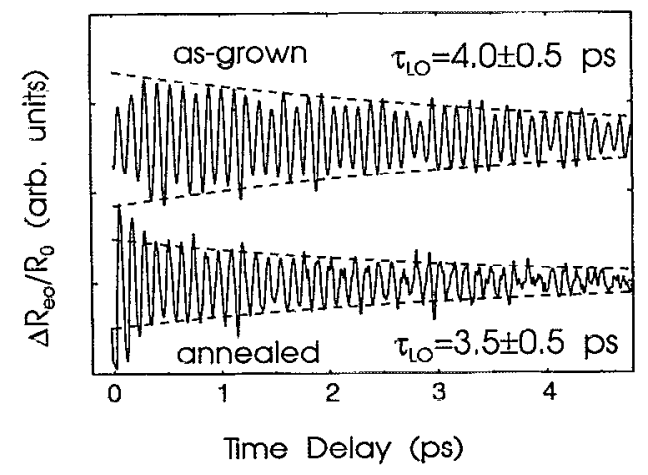

FIG. 3. Time-resolved coherent phonon oscillations in annealed and asgrown LT GaAs. The dashed lines are calculated with the indicated time constants for the decay.

when excess arsenic precipitates into As clusters. The additional fast recombination rate in the annealed sample has to be related to the recombination in As clusters of metallic character. The effect of transient charging of a cluster ${ }^{13}$ is neglected in this interpretation, since the spatial electric field distribution around a cluster contributes with both positive and negative sign to the REOS signals.

The coherent oscillations in as-grown and annealed LT GaAs are depicted in Fig. 3. These spectra are recorded with a modified REOS technique, which is sensitive to the time derivative of the signals only. ${ }^{9}$ The coherent phonons are generated by ultrafast initial field changes on a time scale shorter than the LO phonon period of approximately 113 fs in GaAs. ${ }^{9}$ The LO phonon lifetime and frequency, which both reveal details on the crystalline quality of the samples, can be determined with high accuracy in time domain. The decay of the coherent amplitude is characterized by a dephasing time $\tau_{\text {Lo }}$ composed by pure dephasing and population decay. In previous work on coherent phonons in high quality MBE GaAs an agreement between $\tau_{\text {LO }}$ of approximately $4 \mathrm{ps}$ and the phonon lifetime determined from coherent anti-Stokes Raman spectroscopy has been found. ${ }^{14}$ For LT GaAs, we observe phonon lifetimes comparable to the lifetime observed in high quality GaAs. The influence of optically injected carriers on the phonon lifetime seems to be negligible at exitation densities below the threshold for effective plasmon-phonon coupling. ${ }^{15}$ The dephasing time in the as-grown sample is slightly larger (4.0 ps) than in annealed LT GaAs ( $3.5 \mathrm{ps}$ ), presumably due to a phonon localization effect in the imperfect lattice. Additionally, the decay of the coherent amplitude deviates from a strict monoexponential behavior. This is a result of different frequencies involved leading to a mode beating in time domain. These differences are more clearly observed in the Fourier spectra of the time-resolved oscillations (Fig. 4). The annealed sample exhibits a LO mode centered at $8.78 \mathrm{THz}\left(292.7 \mathrm{~cm}^{-1}\right)$, which is close to the $\Gamma_{15}$ LO phonon frequency in crystalline GaAs. This close correlation confirms crystalline quality of annealed LT GaAs in comparison to high quality MBE GaAs. The spectrum of the as-grown sample exhibits a red shift of the LO phonon $\left(0.09 \mathrm{THz}, 3 \mathrm{~cm}^{-1}\right)$, which is six times larger than the recently reported red shift due to the reduction in the 


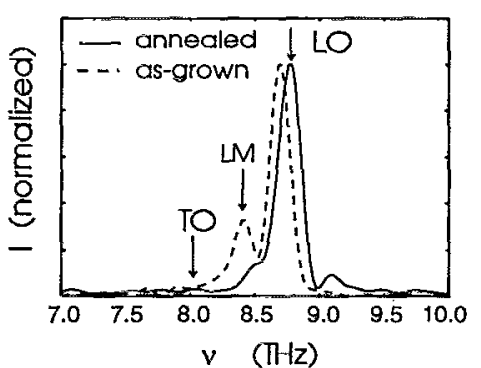

FIG. 4. Fourier spectra of coherent phonon signals from annealed and as-grown LT GaAs. The arrows indicate the spectral position of the TO and $L O$ phonon in crystalline GaAs, and of the local mode (LM) in as-grown LT GaAs.

displacement field of the LO phonon in the presence of high $\mathrm{As}_{\mathrm{Ga}}$ antisite density. ${ }^{16}$ The observed red shift cannot be explained exclusively by the reduction of the displacement field by excess As below 2\%. Therefore, additional tensorial strain has to be assumed for lowering the LO frequency. ${ }^{17}$ Additionally, we observe a mode at $8.4 \mathrm{THz}$ $\left(280 \mathrm{~cm}^{-1}\right)$ in as-grown LT GaAs, which vanishes after annealing. This mode is attributed to a local As-As mode (LM), which has been found also in IR absorption spectra of amorphous arsenic. ${ }^{18}$ The ratio of the $\mathrm{LO}$ intensity to the LM intensity is approximately 3 , which is surprisingly high when the $\mathrm{LM}$ is related to $\mathrm{As}_{\mathrm{Ga}}$ antisites or As interstitials at densities below $10^{20} \mathrm{~cm}^{-3}$. A specific antisiteinterstitial complex, ${ }^{19}$ which disappears after annealing, may also account for the observation of the LM and is in accordance with strain in the as-grown sample. In this context it is interesting to note that in heavily doped LT $\mathrm{GaAs}$ similar complexes account for $\mathrm{LMs} .{ }^{20} \mathrm{~A}$ final assignment of the LM in the vicinity of the TO and LO phonon needs further investigation.

In conclusion we demonstrated a new all-optical method for the time-resolved investigation of LT grown III-V compounds. Reflective electro-optic sampling delivers firm data on specific differences in electric field dynamics, transport properties, carrier lifetimes, and coherently excited LO phonons in as-grown and annealed LT GaAs. The transport dynamics of high resistivity annealed LT GaAs is controlled by a superposition of screening and photo-Dember effect. The recombination of carriers in trapped states is monitored via the evolution of the electric field towards its unperturbed value. In the case of as-grown LT GaAs a strict monoexponential decay of 125 ps is observed due to recombination of carriers trapped at $\mathrm{As}_{\mathrm{Ga}}$ antisites. For annealed LT GaAs the presence of an additional time constant of $7 \mathrm{ps}$ is related to the presence of As precipitates. The analysis of coherent LO phonon dynamics reveals information on structural defects in as-grown LT GaAs.

'T. Motet, J. Nees, S. Williamson, and G. Mourou, Appl. Phys. Lett. 59, 1455 (1991)

${ }^{2}$ A. C. Warren, J. H. Burroughs, J. M. Woodall, D. T. Mcinturff, R. T. Hodgson, and M. R. Melloch, IEEE Electron. Devices Lett. EDL-12, 527 (1991).

${ }^{3}$ M. Klingenstein, J. Kuhl, R. Nötzel, K. Ploog, J. Rosenzweig, C. Moglestue, A. Hülsmann, Jo. Schneider, and K. Köhler, Appl. Phys. Lett. 60, 627 (1992).

${ }^{4}$ F. W. Smith, A. R. Calawa, C.-L. Chen, M. J. Manfra, and L. J Mahoney, IEEE Electron. Devices Lett. 9, 77 (1988).

${ }^{5}$ See, e.g., A. C. Warren, J. M. Wodall, P. D. Kirchner, X. Yin, F. Pollak, M. R. Mclloch, N. Otusaka, and K. Mahalingam, Phys. Rev. B 46, 4617 (1992), and references therein.

${ }^{6}$ X. Q. Zhou, H. M. van Driel, W. W. Rühle, Z. Gogolak, and K. Ploog, Appl. Phys. Lett. 61, 3020 (1992).

${ }^{7}$ S. Gupta, M. Y. Frankel, J. A. Valdmanis, J. F. Whitaker, and G. Mourou, Appl. Phys. Lett. 59, 3276 (1991).

${ }^{8}$ T. Dekorsy, T. Pfeifer, W. Kütt; and H. Kurz, Phys. Rev. B 47, 3842 (1993).

${ }^{9}$ T. Pfeifer, T. Dekorsy, W. Kütt, and H. Kurz, Appl. Phys. A 55, 482 (1992).

${ }^{10}$ T. S. Moss, Optical Properties of Solids (Butterworths Scientific, London, 1959).

${ }^{11}$ M. Kaminska, E. R. Weber, Z. Liliental-Weber, R. Leon, and Z. U. Rek, J. Vac. Sci. Technol. B 7, 710 (1989).

${ }^{12}$ K. P. Korona, M. Kaminska, J. M. Baranowki, and E. R. Weber, Mater. Sci. Eng. B 22, 41 (1993).

${ }^{13}$ A. C. Warren, J. M. Woodall, J. L. Freeouf, D. Grischkowsky, M. R. Melloch, and N. Osuka, Appl. Phys. Lett. 57, 1331 (1990).

${ }^{14}$ F. Vallee and F. Bogani, Phys. Rev. B 43, 12049 (1991).

${ }^{15}$ T. Pfeifer, T. Dekorsy, W. Kütt, and H. Kurz, in Phonon Scattering in Condensed Matter VII, edited by M. Meissner and R. O. Pohl (Springer, Berlin, 1993), p. 110.

${ }^{16}$ T. A. Gant, H. Shen, J. R. Flemish, L. Fotiadis, and M. Dutta, Appl. Phys. Lett. 60, 1453 (1992).

${ }^{17}$ H. S. Jang, H. Y. Cho, S. W. Lee, S. K. Min, I.-S. Yang, and J. Yang, Appl. Phys. A 56, 571 (1993).

${ }^{18}$ R. J. Nemanich, G. Lucovsky, W. Pollard, and J. D. Joannopoulos, Solid State Commun. 26, 137 (1978).

${ }^{19}$ D. J. Chadi, Phys. Rev. B 46, 15053 (1992).

${ }^{20}$ S. A. McQuaid, R. C. Newmann, M. Missous, and S. O'Hagan, J. Cryst. Growth 127, 515 (1993). 